ZU-TH-98/20

SU-GP-98/10-1

gr-qc/9812024

\title{
On the Generality of Refined Algebraic Quantization
}

\author{
Domenico Giulini \\ Institute for Theoretical Physics, CH-8057 Zürich, Switzerland \\ Donald Marolf \\ Physics Department, Syracuse University, Syracuse, New York 13244
}

(November, 1998)

\begin{abstract}
The Dirac quantization 'procedure' for constrained systems is well known to have many subtleties and ambiguities. Within this ill-defined framework, we explore the generality of a particular interpretation of the Dirac procedure known as refined algebraic quantization. We find technical conditions under which refined algebraic quantization can reproduce the general implementation of the Dirac scheme for systems whose constraints form a Lie algebra with structure constants. The main result is that, under appropriate conditions, the choice of an inner product on the physical states is equivalent to the choice of a "rigging map" in refined algebraic quantization.
\end{abstract}




\section{INTRODUCTION}

The Dirac quantization scheme [1] has long been a subject of study by mathematical physicists, by researchers interested in quantum gravity, and by others. Briefly, it suggests that certain equations of motion (the constraints of a gauge system) need not be imposed directly at the operator level, but should be imposed as conditions that select certain 'physical states.' This procedure, however, has a number of difficulties and ambiguities, not least of which arise from the fact that additional input is typically required to define a Hilbert space structure on the physical states. Some progress toward resolving these issues (or at least toward structuring their discussion) has been made in each of the BRST approach (see, for example [2 (4), the Geometric Quantization approach [5], coherent state quantization [6,7], $C^{*}$-algebra methods [8,9], the Algebraic Quantization approach [10 12], the Klein-Gordon style approach (in the context of minisuperspace models of cosmology) 113, and the Refined Algebraic Quantization approach [14,15] (which is closely related to Rieffel Induction [16,17] and other works [18, 19]).

Some researchers will feel that Refined Algebraic Quantization (RAQ) is the most abstract of these attempts. This is to be expected of a truly general approach, and we argue below that RAQ is to some degree universal. Since the Dirac procedure is well known to be rather vaguely defined, we must first spend a certain amount of time motivating and stating a precise version of the Dirac procedure. One of the main inputs that we will make will be to assume that the $*$-algebra $\mathcal{A}_{\text {obs }}$ of observables is fixed and represented on a dense, linear subspace $\Phi$ of some (non-physical) Hilbert space before the constraints are solved. We also argue in section IIA that it is natural to impose a number of technical conditions, the most important of which are that 1 ) the constraints are solved in the space $\Phi^{*}$ of linear functionals on $\Phi, 2)$ the physical Hilbert space $\mathcal{H}_{\text {phys }}$ is in fact contained in the space $\Phi^{*}$, and 3) that the Hilbert space topology of $\mathcal{H}_{\text {phys }}$ is finer than the topology induced on $\mathcal{H}_{\text {phys }}$ by inclusion in $\Phi^{*}$. At the end of section IIA, we state a precise version of the Dirac scheme' within which we can investigate the generality of refined algebraic quantization.

This paper is concerned with the mathematical aspects of constraint quantization. Although it is self-contained, it is not intended to be a review. More introductory treatments of RAQ including a number of worked examples are available in [14, 15. The examples considered in 19 23 may also be helpful. In particular, they illustrate in detail the relevance of RAQ to particular issues in gravitational physics.

Below, we will consider only systems whose constraints form a Lie algebra, as it is only for such systems that RAQ has yet been formulated. We note however that our main motivation, and the original one for RAQ, comes from gravity. The full gravitational constraints form an algebra that involves structure functions as opposed to structure constants, and so is not at present addressed even in principle by RAQ. Our hope is that RAQ can be generalized to

\footnotetext{
${ }^{1}$ Note that we do not claim this to be the only precise formulation of the Dirac scheme.
} 
include cases with structure constants. This is expected to be difficult, due to the difficulties discussed in [24]. However, 25] takes a tantalizing step in this direction in its treatment of non-unimodular Lie groups.

A skeletal discussion of refined algebraic quantization itself is given in section IIB, at which point it is shown that all quantizations produced by RAQ belong to the class described in section IIA. This sets the stage for the discussion of section III, in which the generality of refined algebraic quantization is investigated within the framework of section IIA. It turns out that there are two main stumbling blocks to proving that refined algebraic quantization can reproduce an arbitrary quantization allowed by section IIA. One of these has to do with the domains of unbounded operators. It can be overcome by an extra technical assumption which, unfortunately, is not entirely satisfactory. The status of this assumption is discussed in section IV. The other stumbling block has to do with showing that the full physical Hilbert space can be obtained, and not just a nontrivial subrepresentation. As discussed in section IV, this can be overcome by a physically motivated (but somewhat complicated) assumption. In the end, we do arrive at a set of conditions under which RAQ can reproduce a general implementation of the interpretation of the Dirac procedure that we state in section IIA.

\section{THE SETTING}

In part $\mathrm{A}$ of this section, we review the Dirac procedure and motivate certain choices that will be made in formulating a precise version of this procedure. In part $\mathrm{B}$, we then show that refined algebraic quantization in fact respects these choices. This sets the stage for the discussion of section III, in which we explore the generality of refined algebraic quantization within our interpretation of the Dirac scheme.

\section{A. A precise form of the Dirac procedure.}

Briefly (but not quite as briefly as above), the Dirac scheme introduces constraint operators $C_{i}$ on a linear space $L$ and requires 'physical' states $|\psi\rangle$ to be solutions of the constraints:

$$
C_{i}|\psi\rangle=0
$$

\footnotetext{
${ }^{2}$ There have been suggestions [26] that, when the constraints generate a non-unimodular gauge group, the physical states should not be exactly solutions to the constraints in the sense of (2.1). The suggestion is that $C_{i}|\psi\rangle$ should give $|\psi\rangle$ times a certain complex number that depends on the group and the particular generator $C_{i}$. When the Dirac scheme is interpreted in this way, the argument below continues to hold with minimal modification.
} 
Operators that commute with the constraints are called observables, and these map physical states to physical states. An inner product should be defined on the physical states to make them into a Hilbert space in which the observables act. Below, we will assume that the constraints are 'first-class' in the sense of Dirac [1] and that they form a Lie algebra (i.e., that their commutators close with structure constants as opposed to structure functions).

Now, there has been much discussion of observables in the context of constraint quantization (see [19,27 29] and references therein). Our philosophy is that the $*$-algebra of quantum observables should be fixed and represented on a Hilbert space before the constraints are solved [ Since the $*$-relations of this algebra must be related to the physical inner product, this will give us some modicum of control over the rest of the construction. We assume that the space carrying this (not yet physical) representation is a dense and linear subspace of the so-called auxiliary Hilbert space $\mathcal{H}_{\text {aux }}$. This idea is explicitly shared by RAQ 14, 15. The original Algebraic Quantization [10] requires this *-algebra to be fixed, but not that it be represented on an inner product space at this stage.

Note that we have not required the observable *-algebra to be explicitly constructed in any sense. The difficulties of explicitly constructing observables in complicated gauge theories are well known. It is sufficient that the $*$-algebra be defined implicitly. We also assume that there is some Hilbert space $\mathcal{H}_{\text {aux }}$ associated with $L$ such that observables are linear but not necessarily bounded operators on $\mathcal{H}_{\text {aux }}$. In practice, however, the natural order is usually reversed: one starts with some natural Hilbert space, like the $L^{2}$-space over the unreduced configuration space (containing gauge degrees of freedom), on which the constraints are represented by linear operators. Recalling that the classical constraints are real, it appears naturalf to identify the $C_{i}$ with self-adjoint operators on $\mathcal{H}_{\text {aux }}$ (though generically they will not be bounded). In general, it is impossible to identify $L$ with $\mathcal{H}_{\text {aux }}$. For example, if the spectrum of any given constraint $C_{i}$ is purely continuous, then it has no normalizable eigenvectors, and in particular none with eigenvalue zero. Thus, the Hilbert space itself contains no solutions. What is needed is some more general mathematical structure, and it is natural to take a hint from spectral theory in which 'generalized eigenvectors' (which are not normalizable) can be identified as certain types of distributions (dual states) associated with $\mathcal{H}_{\text {aux }}$. Typical examples of this sort are the constructions of position- and momentum-'eigenstates' in ordinary quantum mechanics. It may of course also happen that zero is simply not part of the whole spectum. In this case normal spectral theory does not offer any solutions. But still this does not yet imply that no 'solutions' to the constraints

\footnotetext{
${ }^{3}$ Note that one cannot simply stop at this point. The constraints contain important physical information and must be imposed, else the full equations of motion will not be satisfied even in the classical limit.

${ }^{4}$ This is certainly the case when the constraints form a Lie algebra. However, when the algebra involves structure functions, taking the $C_{i}$ self-adjoint is in general not consistent (see [24]). This is the main difficulty in generalizing RAQ to systems with structure functions.
} 
exist. Since they require to pick a particular representation of the gauge group generated by the $\left\{C_{i}\right\}$ from among all the representations 'contained' in that on $\mathcal{H}_{\text {aux }}$, one can indeed considerably weaken the notion of 'containment' to solve the constraints in many such problematic cases. This is further explained in [25]. This reference also explains why and how the Dirac condition 2.1 has to be modified in case the group generated by the $\left\{C_{i}\right\}$ is not unimodular, in which case the spectral value zero is not relevant.

Following this suggestion we proceed by looking for a dense linear subspace $\Phi \subset \mathcal{H}_{\text {aux }}$ with the properties that (i) $\Phi$ is contained in the domain of each $C_{i}$, and (ii) $\Phi$ is left invariant (as a set) under the action of each $C_{i}$. In order to define the algebra of observables we now look for the set of all operators $A$ on $\mathcal{H}_{\text {aux }}$ such that (i') the domain of $A$ and $A^{\dagger}$ contains $\Phi$, and (ii') $A$ and $A^{\dagger}$ leave $\Phi$ invariant. This set contains the $C_{i}$ 's and forms a $*$-algebra, $\mathcal{A}$, of linear operators on $\Phi$. Within $\mathcal{A}$ we may consider the commutant $\left\{C_{i}\right\}^{\prime}$, i.e. the set of all $A \in \mathcal{A}$ commuting with every $C_{i}$, which is a $*$-subalgebra. This is essentially our *-algebra of observables, $\mathcal{A}_{\text {obs }}$. Note that $\mathcal{A}_{\text {obs }}$ becomes an algebra only after restriction to $\Phi$. This is because we allowed for unbounded operators so that multiplication as operators on $\mathcal{H}_{\text {aux }}$ makes generally no sense due to mismatches between ranges and domains. The *-operation is that induced by the adjoint operation $\dagger$ for operators on $\mathcal{H}_{\text {aux }}$, which on $\mathcal{A}_{\text {obs }}$ becomes an abstract $*$ operation which we denote by a $\star$. From now on this operation will be the only carrier of information about the inner product of $\mathcal{H}_{\text {aux }}$. We also remark on the fact that the subalgebra generated by the $C_{i}$ might have a nontrivial intersection with $\mathcal{A}_{\text {obs }}$, given by the center $\left\{C_{i}\right\}^{c}:=\left\{C_{i}\right\} \cap\left\{C_{i}\right\}^{\prime}$ of $\left\{C_{i}\right\}$, whose elements annihilate physical states. But physical states are also annihilated by any product of operators in $\mathcal{A}_{\text {obs }}$ which contain at least one member from $\left\{C_{i}\right\}^{c}$, i.e., by the ideal $I$ in $\mathcal{A}_{\text {obs }}$ generated by $\left\{C_{i}\right\}^{c}$. Hence we should eventually identify the algebra of physical observables with the quotient $\mathcal{A}_{\mathrm{obs}} / I$. This being understood, we shall save notation and just consider $\mathcal{A}_{\text {obs }}$.

The purpose of considering $\Phi$ is to introduce the concept of distributional solutions to the constraints. We recall (see, e.g. [30]) that to make the notion of distributional states precise requires the Hilbert space structure of $\mathcal{H}_{\text {aux }}$ and the choice of of some linear subspace $\Phi \subset \mathcal{H}_{\text {aux }}$. The space $\Phi$ often comes equipped with its own intrinsic topology, which is to be distinguished from the one that it inherits from being a subspace of $\mathcal{H}_{\text {aux }}$. The intrinsic topology is usually finer and complete. Here, however, we will simply take $\Phi$ to have the topology induced by inclusion in $\mathcal{H}_{\text {aux }}$. Without loss of generality we took $\Phi$ to be dense in $\mathcal{H}_{\text {aux }}$, as the auxiliary Hilbert space can always be replaced by the closure of $\Phi$.

Naturally associated with $\Phi$ is its algebraic dual, $\Phi^{*}$, given by the space of all linear maps from $\Phi$ into the complex numbers. Here we follow a weaker construction than that of standard spectral analysis, as we use the algebraic dual instead of the topological dual (which

\footnotetext{
${ }^{5}$ Standard examples of Gel'fand-triples in quantum mechanics have as $\Phi$ the space of rapidly decreasing or compactly supported $C^{\infty}$-functions. These spaces are topologized by a complete, nuclear topology.
} 
would contain only continuous maps). The topology of $\Phi^{*}$ is that of pointwise convergence, which means that a sequence $f_{n} \in \Phi^{*}$ converges to $f \in \Phi^{*}$ if and only if $f_{n}(\phi) \rightarrow f(\phi)$ for all $\phi \in \Phi$. The point now is that $\mathcal{H}_{\text {aux }}$ can be naturally identified with a subspace of $\Phi^{*}$, since vectors in $\mathcal{H}_{\text {aux }}$ define linear functionals on the subset $\Phi$ by taking inner products. Hence there is a natural inclusion $j: \mathcal{H}_{\text {aux }} \hookrightarrow \Phi^{*}$.

Here again, the intrinsic topology of $\mathcal{H}_{\text {aux }}$ (given by the Hilbert norm) should be distinguished from that inherited as a subspace of $\Phi^{*}$. The former one is finer so that the inclusion $j$ is continuous. In summary, we have the following triple of spaces and continuous embeddings (with a little abuse of language we may call it a 'Gel'fand triple')

$$
\Phi \stackrel{i}{\hookrightarrow} \mathcal{H}_{\mathrm{aux}} \stackrel{j}{\rightarrow} \Phi^{*}
$$

Depending on the choice of $\Phi, \Phi^{*}$ should be thought of as 'a controlled enlargement' of $\mathcal{H}_{\text {aux }}$. Technically, the enlargement may be understood as completion in a coarser topology. It is now the space of distributional states $\Phi^{*}$ that we identify with $L$ and amongst which we seek the solutions to the constraints. If zero is not in the discrete part of the spectrum of all $C_{i}$, solutions must be sought in the complement of $\mathcal{H}_{\text {aux }}$ in $\Phi^{*}$. We do not address here the interesting question of how the choice of $\Phi$ is to be made in general but merely note that some scheme along the lines outlined above appears necessary for the implementation of our philosophy. We strongly suspect that the choice of $\Phi$ requires some form of physical (and not just mathematical) input. See [15] for some discussion on this subject, with examples.

Having established the various spaces, we next need to understand the action of operators. Since the constraints preserve $\Phi$ they have a dual action on $\Phi^{*}$ (displayed in (2.4)) and we may discuss distributional solutions $f \in \Phi^{*}$, defined by

$$
f(C \phi)=0 \quad \text { for all } \phi \in \Phi
$$

Recall that the observable algebra should act on the space of solutions. Clearly $\mathcal{A}_{\text {obs }}$ acts on $L$ since an operator $\mathcal{O}$ on $\mathcal{H}_{\text {aux }}$ has a natural action on $L=\Phi^{*}$ if and only if its adjoint $\mathcal{O}^{\dagger}$ maps the space $\Phi$ into itself. In this case, $\mathcal{O}$ can take any $f \in \Phi^{*}$ to $\mathcal{O} f$, defined by the dual action of $\mathcal{O}$ on $\Phi$ :

$$
(\mathcal{O} f)(\phi):=f\left(\mathcal{O}^{\dagger} \phi\right) \quad \text { for all } \phi \in \Phi
$$

where for $\mathcal{O} \in \mathcal{A}_{\text {obs }}$ we should write $\star$ instead of $\dagger$. It is due to the adjoint (i.e., $\left.\star\right)$ in (2.4) that $\Phi^{*}$ will carry an anti-linear representation of $\mathcal{A}_{\mathrm{obs}}$.

The final issues have to do with the topology on $\Phi^{*}$ and the construction of the physical Hilbert space $\mathcal{H}_{\text {phys }}$. The most general construction one could imagine would be to identify some subspace in the space $\mathcal{V} \subset \Phi^{*}$ of all solutions to (2.1) and make it into a pre-Hilbert space by introducing an inner product. One would then complete this to a Hilbert space $\mathcal{H}_{\text {phys }}$. This completion, however, could result in a vastly larger physical Hilbert space. To control this, we wish every physical state to represent a genuine solution in the sense of (2.3), which means that we require $\mathcal{H}_{\text {phys }} \subseteq \mathcal{V}$. Note that the identification of $\mathcal{H}_{\text {phys }}$ with a 
subset of $\mathcal{V}$ is on the level of sets only. The topology on $\mathcal{H}_{\text {phys }}$ is that of norm convergence and not the subspace topology induced by $\Phi^{*}$. In particular, in the former topology $\mathcal{H}_{\text {phys }}$ must be complete. Hence it is natural to assume that the Hilbert space topology be finer than that induced by $\Phi^{*}$, that is, if $f_{n}$ converges to $f$ in $\mathcal{H}$, then $f_{n}$ converges to $f$ in $\Phi^{*}$ as well.

The observables should act via an (anti-)*-representation on $\mathcal{H}_{\text {phys }}$. If $\mathcal{H}_{\text {phys }}$ were all of $\mathcal{V}$, and not just a subspace, then each operator together with its adjoint would be defined everywhere on $\mathcal{H}_{\text {phys }}$ and hence necessarily be bounded. In this case we would have obtained an (anti-)*-representation of $\mathcal{A}_{\text {obs }}$ into the $*$-algebra of bounded operators. Presently it is not obvious to us what precisely the technical restrictions are that would ensure the possibility to turn all of $\mathcal{V}$ into the physical Hilbert space, and whether appropriate adjustments in one's choice of $\Phi$ could lead to such a possibility. Hence we proceed by admitting that $\mathcal{A}_{\text {obs }}$ is generally represented by unbounded operators, which means that there is also no reason for the observables to preserve $\mathcal{H}_{\text {phys }}$. This implies that we must take care to state in just what sense this algebra is represented on $\mathcal{H}_{\text {phys }}$. We proceed in analogy with our treatment of $\mathcal{A}_{\text {obs }}$ on $\mathcal{H}_{\text {aux }}$. That is, we assume that there is some dense subspace $\Phi_{\text {phys }} \subset \mathcal{H}_{\text {phys }}$ such that $\Phi_{\text {phys }}$ is a common invariant domain for all operators $\mathcal{O}$ in $\mathcal{A}_{\text {obs. }}$. This allows the algebraic relations to be represented in the action of $\mathcal{A}_{\text {obs }}$ on $\Phi_{\text {phys }}$. We also require that the star relations be represented in the sense that, for any $\phi_{1}, \phi_{2} \in \Phi_{\text {phys }}$ and any $\mathcal{O} \in \mathcal{A}_{\text {obs }}$, we have

$$
\left(\phi_{1}, \mathcal{O} \phi_{2}\right)=\overline{\left(\phi_{2}, \mathcal{O}^{\star} \phi_{1}\right)}
$$

where $\star$ represents the $*$-operation in the algebra. In this sense, the subspace $\Phi_{\text {phys }} \subset \mathcal{H}_{\text {phys }}$ carries a star representation of the observable algebra. By construction this automatically holds if $\mathcal{H}_{\text {phys }}=\mathcal{V}$, and is put as an extra requirement on $\mathcal{H}_{\text {phys }}$ if it is chosen as a proper subspace.

Following the idea that the $*$-algebra of observables is derived from operators of an auxiliary Hilbert space and fixed before the constraints are solved has now led us to a welldefined setting in which to discuss the Dirac procedure. We have a Hilbert space $\mathcal{H}_{\text {aux }}$, a set of self-adjoint constraint operators $C_{i}$, and a subspace $\Phi$ which is mapped to itself by the constraints. Without loss of generality, we may take $\Phi$ to be dense in $\mathcal{H}_{\text {aux }}$. The observable algebra $\mathcal{A}_{\text {obs }}$ is defined by the set of operators on $\mathcal{H}_{\text {aux }}$ which, after restriction to $\Phi$, commute with the constraints and map $\Phi$ to itself. We seek a Hilbert space $\mathcal{H}_{\text {phys }}$ which can be identified (as a vector space representation of $\mathcal{A}_{\text {obs }}$ ) with a subspace of $\Phi^{*}$, the algebraic dual to $\Phi$. Under this identification, states in $\mathcal{H}_{\text {phys }}$ are annihilated by the (dual) action of the constraints on $\Phi^{*}$, and a dense subspace $\Phi_{\text {phys }}$ carries an (anti)-*-representation of the observable algebra given by its dual action on $\Phi^{*}$. Finally, the Hilbert space topology of $\mathcal{H}_{\text {phys }}$ must be finer than the topology on $\Phi^{*}$. 


\section{B. Refined Algebraic Quantization}

Let us now take a moment to recall what is meant by refined algebraic quantization, and to show that it fits into the precise form of the Dirac procedure stated above. First, we recall [14, 15] that Refined Algebraic Quantization requires a choice of $\mathcal{H}_{\text {aux }}, C_{i}$, and $\Phi$ and gives a definition of $\mathcal{A}_{\text {obs }}$ in exactly the same manner as section IIA. The main point of RAQ, however, is that the inner product is defined by a so-called 'rigging map' $\eta$ from $\Phi$ to $\Phi^{*}$ through

$$
\left(\eta\left(\phi_{1}\right), \eta\left(\phi_{2}\right)\right)_{\mathrm{phys}}:=\phi_{1}\left[\eta\left(\phi_{2}\right)\right]:=\eta\left(\phi_{2}\right)\left[\phi_{1}\right],
$$

where the physical Hilbert space, $\mathcal{H}_{\text {phys }}^{\prime}$, is now defined by taking the closure of the image of $\eta$ in this inner product. Alternatively, one may read (2.6) as a semi-definite inner product on $\Phi$ and identify $\mathcal{H}_{\text {phys }}^{\prime}$ with the closure of $\Phi / \operatorname{kernel}(\eta)$. The rigging map is required to satisfy certain properties (it must be 'real', 'positive', and 'symmetric') that are equivalent to the inner product (2.6) being Hermitian.

The rigging map must also intertwine the representations of the observable algebra on $\Phi$ and $\Phi^{*}$. In [14,15], this last property was called 'commuting' with the observable algebra. This means that, for any $\mathcal{O} \in \mathcal{A}_{\text {phys }}$ and any $\phi \in \Phi$, we have

$$
\mathcal{O}(\eta \phi)=\eta(\mathcal{O} \phi)
$$

Note that this implies that the image of $\eta$ (all of which lies in $\mathcal{H}_{\text {phys }}^{\prime}$ ) is an invariant domain for the action of $\mathcal{A}_{\text {obs }}$ on $\mathcal{H}_{\text {phys }}^{\prime}$. Thus, we may take the image of $\eta$ to be the space $\Phi_{\text {phys }}$ required by the formulation of the Dirac procedure stated at the end of section IIA.

We would like to show that refined algebraic quantization in fact satisfies all requirements of our version of the Dirac procedure. At this point, it is clear that the requirements for $\mathcal{H}_{\text {aux }}$, $C_{i}, \Phi$, and $\mathcal{A}_{\text {obs }}$ are satisfied, but we must still check that, in RAQ, $\mathcal{H}_{\text {phys }}^{\prime}$ may be considered a subspace of $\Phi^{*}$ with a finer topology. To verify this, consider the map $\sigma: \mathcal{H}_{\text {phys }}^{\prime} \rightarrow \Phi^{*}$ defined for $f \in \mathcal{H}_{\text {phys }}^{\prime}$ by

$$
(\sigma f)[\phi]=(f, \eta(\phi))_{\text {phys }} .
$$

We first note that $\sigma f$ vanishes only if $f$ is orthogonal to all states in the image of $\eta$. But this image is dense in $\mathcal{H}_{\text {phys }}^{\prime}$ by construction, so $\sigma$ is an embedding of linear spaces. Moreover, $\sigma$ is continuous since the inner product (2.8) is continuous in $f$. Thus, we may use $\sigma$ to identify $\mathcal{H}_{\text {phys }}^{\prime}$ with a subspace of $\Phi^{*}$ and we see that the Hilbert space topology of $\mathcal{H}_{\text {phys }}^{\prime}$ is finer than the topology induced by this inclusion. Moreover, since $\eta$ commutes with the action of $\mathcal{A}_{\mathrm{obs}}$, we have

$$
\begin{aligned}
(\sigma \mathcal{O} f)[\phi] & =\left(f, \eta\left(\mathcal{O}^{\dagger} \phi\right)\right)_{\mathrm{phys}} \\
& =(\sigma f)\left[\mathcal{O}^{\dagger} \phi\right] \\
& =(\mathcal{O} \sigma f)[\phi]
\end{aligned}
$$

Thus, $\sigma:$ Image $(\eta) \rightarrow$ Image $(\sigma)$ is an isomorphism of vector space representations of $\mathcal{A}_{\text {obs. }}$ If follows that refined algebraic quantization does in fact satisfy all of the requirements stated at the end of section IIA. 


\section{THE GENERALITY OF REFINED ALGEBRAIC QUANTIZATION}

The last paragraph in section IIA gives a precise statement of the Dirac prescription but, a priori, there may still be a great many distinct ways to construct appropriate physical Hilbert spaces, which may or may not be related to RAQ. However, we now show that our version of the Dirac procedure (from IIA) provides at least a natural candidate for a rigging map. Whether or not it actually is a rigging map depends on certain technical conditions involving the domains of operators. In the case that this condition is satisfied, it will follow that refined algebraic quantization can construct at least a nontrivial subrepresentation of the original representation of $\mathcal{A}_{\text {obs }}$ on $\mathcal{H}_{\text {phys }}$. Under a further technical condition, the entire representation of $\mathcal{A}_{\text {obs }}$ on $\mathcal{H}_{\text {phys }}$ can be constructed through RAQ.

As observed in section IIB, refined algebraic quantization and section IIA both involve a choice of $\mathcal{H}_{\text {aux }}, C_{i}$, and $\Phi$, and the restrictions on this choice are the same in both cases. They then use identical definitions of $\mathcal{A}_{\text {obs }}$. However, section IIA places only a few restrictions on the physical Hilbert space $\mathcal{H}_{\text {phys }}$, while refined algebraic quantization requires that the physical Hilbert space be constructed by a certain method involving a rigging map. Thus, our task is to investigate whether a general physical inner product satisfying the conditions of section IIA can in fact be constructed through a rigging map as would be required by refined algebraic quantization.

To do this, consider any $\phi \in \Phi$. Since $\mathcal{H}_{\text {phys }} \subset \Phi^{*}$ and since convergence in $\mathcal{H}_{\text {phys }}$ implies convergence in $\Phi^{*}, \phi$ defines a continuous linear functional on $\mathcal{H}_{\text {phys }}$. But $\mathcal{H}_{\text {phys }}$ is a Hilbert space, which means that there is some $\phi_{0} \in \mathcal{H}_{\text {phys }}$ such that, for all $f \in \mathcal{H}_{\text {phys }}, \phi(f)=\left(\phi_{0}, f\right)$. Thus, we may introduce a map $\eta: \Phi \rightarrow \mathcal{H}_{\text {phys }}$ given by $\eta(\phi)=\phi_{0}$.

The map $\eta$ is anti-linear, and the inner product on $\mathcal{H}_{\text {phys }}$, restricted to Image $(\eta)$, is

$$
\left(\eta\left(\phi_{1}\right), \eta\left(\phi_{2}\right)\right)_{\mathrm{phys}}=\phi_{1}\left[\eta\left(\phi_{2}\right)\right]=\eta\left(\phi_{2}\right)\left[\phi_{1}\right]
$$

If $\eta$ is a rigging map, this is just the Refined Algebraic inner product. Note that since the inner product on $\mathcal{H}_{\text {phys }}$ is Hermitian, $\eta$ is real, positive, and symmetric (in the sense of Refined Algebraic Quantization).

Now, it remains only to investigate the behavior of $\eta$ with respect to the observables. Here, a difficulty arises involving the domains of the unbounded operators in $\mathcal{A}_{\text {obs }}$. Let $\Phi^{\prime}$ phys be the image of $\eta$ in $\mathcal{H}_{\text {phys }}$. Refined algebraic quantization requires that $\Phi^{\prime}$ phys carry a $*$-representation of $\mathcal{A}_{\text {obs }}$. However, section IIA in no way restricts the choice of which subspace should carry such a *-representation, and in particular does not appear to restrict the domains of the operators in any way. Thus, it does not appear necessary that $\Phi^{\prime}{ }_{\text {phys }}$ even overlap the domain of any observable $\mathcal{O}$. Note that this would not be an issue if we could somehow arrange to work with bounded operators on the physical Hilbert space, as in the case where $\mathcal{H}_{\text {phys }}=\mathcal{V}$.

Thus, in order to proceed, we must introduce the additional assumption that the image $\Phi_{\text {phys }}^{\prime}$ of $\eta$ is contained in the dense subspace $\Phi_{\text {phys }}$. In this case, we can can show that $\eta$ satisfies the last requirement of a rigging map. Namely, it commutes with the action of 
observables. To see this, consider any $\phi_{1}, \phi_{2} \in \Phi$ and $\mathcal{O} \in \mathcal{A}_{\text {phys. }}$ We can use (3.1), the hermiticity of the physical inner product, and the definition of the dual action to conclude that

$$
\eta\left(\mathcal{O} \phi_{2}\right)\left[\phi_{1}\right]=\overline{\eta\left(\phi_{1}\right)\left[\mathcal{O} \phi_{2}\right]}=\overline{\mathcal{O}^{\star} \eta\left(\phi_{1}\right)\left[\phi_{2}\right]}
$$

But this is the (complex conjugate of) the inner product $\left(\eta \phi_{2}, \mathcal{O}^{\star} \eta\left(\phi_{1}\right)\right)_{\text {phys }}$ on the physical Hilbert space. Now, using the fact that $\Phi_{\text {phys }}$ carries a $*$ representation of the observables, we can rewrite the right-hand side above as $\left(\eta \phi_{1}, \mathcal{O} \eta\left(\phi_{2}\right)\right)_{\text {phys }}=\left(\mathcal{O} \eta\left(\phi_{2}\right)\right)\left[\phi_{1}\right]$. Thus, the observables do indeed commute with the map $\eta$ and it is a rigging map. The closure of the image of $\eta$ in $\mathcal{H}_{\text {phys }}$ is the corresponding Hilbert space $\mathcal{H}_{\text {phys }}^{\prime}$ defined by RAQ. Note that since $\mathcal{A}_{\text {obs }}$ maps $\Phi$ into itself, it must also preserve the image of $\eta$.

Now consider any nonzero $f \in \mathcal{H}_{\text {phys }}$. Then, clearly, there exists some $\phi \in \Phi$ for which $f(\phi)$ and hence $\eta(\phi)$ is non-zero. Thus, the image of $\eta$ is not trivial unless $\mathcal{H}_{\text {phys }}=\{0\}$.

It follows that, given our extra condition about the containment of $\Phi_{\text {phys }}^{\prime}$ in $\Phi_{\text {phys }}$, at least a nontrivial subrepresentation of $\mathcal{A}_{\text {obs }}$ on $\mathcal{H}_{\text {phys }}$ can be constructed by Refined Algebraic Quantization for any implementation of the Dirac scheme satisfying the requirements of section IIA. If $\Phi_{\text {phys }}^{\prime}$ is dense in $\mathcal{H}_{\text {phys }}$, then RAQ can construct the entire representation. Requiring this space to be dense in $\mathcal{H}_{\text {phys }}$ may be natural from a physical point of view as it guarantees that, in some sense, any state in $\mathcal{H}_{\text {phys }}$ can be approximated arbitrarily well by a state in the auxiliary space

\section{DISCUSSION}

For systems whose constraints form a Lie algebra, we have shown that RAQ is, to some degree, universal. However, we have introduced a number of technical conditions whose physical meaning is unclear, and we have arrived only at the conclusion that RAQ is capable of constructing a nontrivial subrepresentation of the physical algebra.

One might expect that one could dispense with some of the technical conditions by working with bounded operators instead of unbounded ones. While this is true to some extent, our current understanding is that the main effect would simply be to replace the technical conditions above with technical conditions of other sorts. The main point here is that we have found no natural way to ensure that some topology (or norm) placed on the observable algebra at the level of $\mathcal{H}_{\text {aux }}$ survives in any way to the level of $\mathcal{H}_{\text {phys }}$. Thus, restricting the observable algebra to bounded operators on $\mathcal{H}_{\text {aux }}$ does not appear to guarantee (in, for example, refined algebraic quantization) that this algebra will be represented by bounded operators on $\mathcal{H}_{\text {phys }}$. In particular, the powerful theorems concerning $C *$-algebras do not apply here, as the restriction that $\mathcal{A}_{\text {obs }}$ leave $\Phi$ invariant leads us to expect that

\footnotetext{
${ }^{6}$ In fact, it seems natural to require that $\Phi$ approximate $\mathcal{H}_{\text {phys }}$ in the sense of states on (perhaps $C *$-subalgebras of) $\mathcal{A}_{\text {obs }}$. The consequences of such a requirement will be left for future studies.
} 
$\mathcal{A}_{\text {obs }}$ is not complete. Requiring by fiat that the entire algebra $\mathcal{A}_{\text {obs }}$ be represented by bounded operators on $\mathcal{H}_{\text {phys }}$ is a strong restriction, and may be too strong to be physically acceptable. An example will be presented in [25] in the context of non-unimodular groups where it is argued that the physically correct approach maps certain operators on $\mathcal{H}_{\text {aux }}$ to operators with a larger norm on $\mathcal{H}_{\text {phys }}$. In particular, it will map a unitary representation $U(g)$ of the gauge group to the (non-unitary) representation $\Delta^{1 / 2}(g)$ given by the modular homomorphism $\Delta: G \rightarrow \mathbf{R}^{+}$. Thus, for the moment at least, we feel constrained to work with unbounded operators.

Now, as stated above, even under all of these technical conditions we have only been able to show that RAQ can reproduce a nontrivial subrepresentation of $\mathcal{A}_{\text {obs }}$ on $\mathcal{H}_{\text {phys }}$. However, we will now show that the introduction of a further assumption guarantees that RAQ can reproduce the entire representation of the observables. While this new assumption is a bit awkward to state, we will see that it follows from a physical motivation.

Suppose that $\mathcal{H}_{\text {phys }}=\mathcal{H}_{1} \oplus \mathcal{H}_{2}$. Recall that if $\mathcal{H}_{1}$ separately reduces [i.e., is invariant under] $\mathcal{A}_{\text {obs }}$, then so does $\mathcal{H}_{2}$. Hence any matrix element of an operator in $\mathcal{A}_{\text {obs }}$ between a state from $\mathcal{H}_{1}$ and a state from $\mathcal{H}_{2}$ necessarily vanishes, so that there is no interference between states in $\mathcal{H}_{1}$ and $\mathcal{H}_{2}$ with respect to $\mathcal{A}_{\text {obs }}$. One says [31] that there is a superselection rule between the two sectors $\mathcal{H}_{1}$ and $\mathcal{H}_{2}$. Pure physical states must therefore lie either in $\mathcal{H}_{1}$ or $\mathcal{H}_{2}$, which form physical Hilbert spaces in their own right.]

Now, it may happen that such a superselection rule arises in a physical Hilbert space $\mathcal{H}_{\text {phys }}$ constructed along the lines of section II. Having constructed the direct sum $\mathcal{H}_{1} \oplus \mathcal{H}_{2}$ in this way, it is tempting to try to construct each sector separately following the Dirac approach. However, it is not clear that this is in general possible. Suppose then that the sector $\mathcal{H}_{2}$ cannot be constructed in isolation while $\mathcal{H}_{1}$ can. If one is to insist upon a Dirac approach, then it is natural to consider $\mathcal{H}_{2}$ less physical than $\mathcal{H}_{1}$.

We may therefore wish to add the extra physical assumption that every superselected sector can be constructed separately by a Dirac procedure as defined in section II. Note that this condition is satisfied by RAQ, as one may construct $\mathcal{H}_{2}$ simply by replacing $\Phi$ by the subspace $\Phi_{2}$ that is mapped into $\mathcal{H}_{2}$ by the rigging map. This new assumption will in fact be sufficient to show that the entire physical Hilbert space $\mathcal{H}_{\text {phys }}$ can be constructed from RAQ. To see this, simply consider the direct sum $\mathcal{H}_{\Sigma}$ of all sectors in $\mathcal{H}_{\text {phys }}$ which can be constructed through RAQ. $\mathcal{H}_{\Sigma}$ carries a representation of $\mathcal{A}_{\text {obs }}$, so there is a superselection rule between $\mathcal{H}_{\Sigma}$ and its orthogonal complement. Now, by our new assumption, this orthogonal complement can be constructed separately following a Dirac procedure. But if this complement is nontrivial, then by our earlier result it must contain some nontrivial

\footnotetext{
${ }^{7}$ A superposition of a vector in $\mathcal{H}_{1}$ with a vector in $\mathcal{H}_{2}$ still defines a physical state, in the sense that it defines a positive linear functional on $\mathcal{A}_{\text {obs }}$. But because of the absence of interference terms, this functional is the same as the mixture of the corresponding individual density matrices. Hence it is a mixed physical state.
} 
subrepresentation constructible by RAQ. Since this is false, we must have $\mathcal{H}_{\Sigma}=\mathcal{H}_{\text {phys }}$.

Thus, the choices available in the Dirac scheme are perhaps not as large as might be thought. Although the final results are not as conclusive as one might like, we have managed to shed light on the class of implementations of the Dirac procedure which can be obtained by refined algebraic quantization. This will be useful in interpreting the strength of results that can be derived within the mathematically powerful setting of refined algebraic quantization. In particular, this will provide a useful perspective on the result to be derived in [25]. That work also considers systems whose constraints form a Lie algebra and shows that, when they converge properly, group averaging techniques [14,18] give the unique implementation of the Dirac quantization scheme in the above sense.

\section{ACKNOWLEDGMENTS}

The authors wish to thank Jim Hartle for prompting them to consider the generality of Refined Algebraic Quantization. DM was supported in part by NSF grant PHY97-22362 and by funds provided by Syracuse University. DG by the Albert-Einstein-Institute, the Swiss National Science Foundation and the Tomalla Foundation. 


\section{REFERENCES}

[1] Dirac, P A M Lectures on Quantum Mechanics (New York: Belfer Graduate School of Science, Yeshiva University, 1964).

[2] C. Becchi, A. Rouet, and R. Stora Comm. Math. Phys. 42 (1975) 127.

[3] I. V. Tyutin. Lebedev preprint FIAN ${ }^{\circ} 39$ (1975).

[4] M. Henneaux and C. Teitelboim Quantization of Gauge Systems (Princeton University Press, Princeton, 1992).

[5] N. Woodhouse, Geometric Quantization, (Oxford University Press, Oxford, 1980).

[6] J. Klauder, "Product Representations and the Quantization of Constrained Systems", to appear in the Fadeev Festschrift; quant-ph/9811051.

[7] J. Klauder, "Coherent State Path Integrals at (Nearly) 40", to appear in the proceedings of the Florence Path Integral Conference; quant-ph/9810043.

[8] H. Grundling, C.A. Hurst, "Algebraic quantization of systems with a gauge degeneracy", Commun. Math. Phys. 98 369-390 (1985).

[9] H. Grundling, Lledó, "Local quantum constraints"; math-ph/9812022.

[10] A. Ashtekar Lectures on Non-perturbative Canonical Gravity (New Jersey: World Scientific, 1991).

[11] A. Ashtekar and R. Tate, J. Math. Phys. 35 (1994) 6434, gr-qc/9405073.

[12] G. Mena Marugán, J. Math. Phys. 37 (1996) 196-205, gr-qc/9506038.

[13] R. Wald Phys. Rev. D 48 (1993) R2377; A. Higuchi and R. Wald, Phys. Rev. D 51 (1995) 544, gr-qc/9407038.

[14] A. Ashtekar, J. Lewandowski, D. Marolf, J. Mourão, and T. Thiemann, J. Math. Phys. 366456 (1995), gr-qc/9504018.

[15] D. Marolf, in Symplectic Singularities and Geometry of Gauge Fields, (Banach Center Publications, Polish Academy of Sciences, Institute of Mathematics Warsaw, 1997); gr-qc/9508015.

[16] N. Landsman, J. Geom. Phys. 15 (1995) 285-319, hep-th/9305088.

[17] M. A. Rieffel, Adv. Math. 13, 176-257 (1974); "Proper actions of groups on C*-algebras" in Mappings of Operator Algebras. Ed. by H. Araki and R. V. Kadison. (Boston, Birkhäuser, 1990) 141-182.

[18] A. Higuchi, Class. Quant. Grav. 8 (1991) 1983.

[19] D. Marolf, Class. Quant. Grav. 12 (1995) 1199, gr-qc/9404053.

[20] D. Marolf, Class. Quant. Grav. 12 (1995) 1441, gr-qc/9409049.

[21] D. Marolf, Class.Quant.Grav. 12 (1995) 2469-2486, gr-qc/9412016.

[22] D. Marolf, Phys. Rev. D53 (1996) 6979-6990, gr-qc/9602019.

[23] J. Louko and C. Rovelli, in preparation.

[24] K. Kuchař Phys. Rev. D 34 (1986) 3031; Phys. Rev. D 34 (1986) 3044.

[25] D. Giulini and D. Marolf, gr-qc/9902045.

[26] G. M. Tuynman, J. Math. Phys. 31 (1990) 83.; C. Duval, J. Elhadad, M. J. Gotay, and G. M. Tuynman in Proceedings of the CRM Workshop on Hamiltonian Systems, 
Transformation Groups, and Spectral Transform Methods, ed. by J. Harnad and J. E. Marsden, (Les Publications du CRM, Montréal, 1990).

[27] B. DeWitt, "The Quantization of Geometry," in Gravitation: An Introduction to Current Research ed. Witten L (New York: Wiley, 1962).

[28] K. Kuchař Time and Interpretations of Quantum Gravity in Proceedings of the 4th Canadian Conference on General Relativity and Relativistic Astrophysics ed. Kunstatter G, Vincent D E, and Williams J G (New Jersey: World Scientific, 1992).

[29] C.J. Isham in Florence 1995, General relativity and gravitation ed. by M. Francaviglia et al, (World Scientific, New Jersey, 1997) pg. 167-209, gr-qc/9510063.

[30] A. Bohm, Quantum Mechanics: Foundations and Applications (Springer, New York, 1986) pg. 100.

[31] N.N. Bogoliubov, A.A. Logunov, A.I. Oksak, I.T. Todorov, General Principles of Quantum Field Theory (Kluwer, Dordrecht, 1990), ch. 6.2. 